\title{
Relationship between the introduced and indigenous parasitoids Torymus sinensis and T. beneficus (Hymenoptera: Torymidae) as inferred from mt-DNA (COI) sequences
}

\author{
Kaori YARA* \\ National Institute for Agro-Environmental Sciences; Ibaraki 305-8604, Japan \\ (Received 16 December 2002; Accepted 17 March 2004)
}

\begin{abstract}
The parasitoid wasps Torymus sinensis (Ts) and three strains of T. beneficus (early-spring strain: TbE; late-spring strain: $\mathrm{TbL}$; autumn strain: $\mathrm{TbA}$ ) are respectively introduced and indigenous natural enemies of the chestnut gall wasp Dryocosmus kuriphilus, an invasive chestnut pest. In this study, the genetic relationships among Ts and three strains of $\mathrm{Tb}$ from Japan, China and Korea were investigated by sequencing a portion of the mitochondrial cytochrome oxidase subunit I (COI) gene. Three distinct groups were recognized in a constructed phylogenetic tree, the first group further consists of three subgroups. Ts belongs to two subgroups, whereas TbL belongs to the remaining subgroup. TbE and $\mathrm{TbA}$ belong to the second group, indicating that $\mathrm{TbL}$ is genetically more closely related to $\mathrm{Ts}$ a different species than to $\mathrm{TbE}$ and $\mathrm{TbA}$ strains considered the same species. This requires a reconsideration, especially for TbL, of their taxonomical treatment. The third group consisted of an indigenous Torymus parasitoid that we were unable to identify morphologically, indicating that an unknown Torymus parasitoid that is both genetically and morphologically different from the Ts and the Tb strains exploits D. kuriphilus in Japan.
\end{abstract}

Key words: Torymus sinensis; Torymus beneficus; cytochrome oxidase subunit I gene (COI); molecular phylogeny

\section{INTRODUCTION}

The parasitoid wasps Torymus sinensis Kamijo (abbreviated Ts) and T. beneficus Yasumatsu et Kamijo $(\mathrm{Tb})$ are, respectively, introduced and indigenous natural enemies of the chestnut gall wasp Dryocosmus kuriphilus Yasumatsu, an invasive chestnut pest. Ts from Hebei Province in China was first released in Kumamoto and Ibaraki Prefectures in 1982 (Murakami, 1997) and has subsequently been released at various other sites in Japan (Shirai et al., 1999). It has up to now been believed that there are three $\mathrm{Tb}$ strains that have different emergence periods (Ôtake, 1987; Murakami, 1981, 1988): tentatively designated in this study as the "early-spring strain" (abbreviated $\mathrm{TbE}$ ), the "late-spring strain" ( $\mathrm{TbL}$ ), and the "autumn strain" (TbA). Other than their emergence periods, little ecological information is available on the three strains of $\mathrm{Tb}$. The adults of $\mathrm{Ts}$ and $\mathrm{Tb}$ have similar characteristics in external morpholo- gies and the males are indistinguishable (Ôtake, 1987). Only the adult females of the two species can be discriminated on the basis of the ratio of the ovipositor sheath length to the thorax length $(\mathrm{O} / \mathrm{T}$ ratio) (Ôtake, 1987). Ts has a larger O/T ratio than $\mathrm{Tb}$ (Moriya et al., 1992).

Although approximately 480 Torymus species have been described throughout the world (Grissell, 1995), their systematics and ecological traits, such as host species and emergence period are not well understood. Further, there are some unknown Torymus parasitoids morphologically unidentified. For example, some Torymus parasitoids collected from galls of $D$. kuriphilus in central Japan could not be categorized as $\mathrm{Ts}$ or $\mathrm{Tb}$ on the basis of the $\mathrm{O} / \mathrm{T}$ ratio (Yara, unpublished data). However, few attempts have been made to identify these morphologically unknown Torymus parasitoids in chestnut trees (Yara et al., 2000).

In addition to the $\mathrm{O} / \mathrm{T}$ ratio, an isozyme marker (malic enzyme) has been used to discriminate be-

*E-mail: yara@niaes.affrc.go.jp

DOI: 10.1303/aez.2004.427 
tween Ts and Tb (Izawa et al., 1992, 1995; Toda et al., 2000; Yara et al., 2000). None of the isozyme markers, including malic enzyme, however, can discriminate between Ts and TbL (Izawa et al., 1996; Shirai et al., 1999). Recently, many insect species have been analyzed using DNA sequence data and genetic and phylogenetic relationships among species-groups of morphologically close species have been revealed (Caterino et al., 2000).

In this study, Torymus parasitoids including the three $\mathrm{Tb}$ strains, Ts and undetermined Torymus parasitoids were collected from galls of $D . k u$ riphilus in Japan, China and Korea. A portion of the mitochondrial cytochrome oxidase subunit I (COI) gene of each parasitoid was sequenced and a phylogenetic tree constructed in order to examine the genetic relationships between $\mathrm{Ts}$ and indigenous Torymus parasitoids, including the three $\mathrm{Tb}$ strains. Furthermore, using the molecular phylogenetic tree, I attempted to determine the phylogenetic position of Torymus parasitoids that have not been classified as either $\mathrm{Ts}$ or $\mathrm{Tb}$ on the basis of the $\mathrm{O} / \mathrm{T}$ ratio.

\section{MATERIALS AND METHODS}

Insect collection. Seventy-seven adult females of Torymus parasitoids originating from 13 sites in Japan, Korea, and China were used for analysis (Fig. 1, Table 1). All the parasitoids, except for Torymus sp. 1 used as an outgroup, were collected from D. kuriphilus galls. Specimens were preserved in a freezer at -30 or $-70^{\circ} \mathrm{C}$ until DNA extraction. To exclude the possibility of hybridization between indigenous Torymus parasitoids and introduced Ts originating in China (Moriya et al., 1992; Izawa et al., 1995), indigenous Torymus parasitoids were collected before introduced Ts became distributed at the collection sites in Japan (Moriya et al., 1992; Murakami, 1997; Shirai et al., 1999). For the same reason, introduced Ts was not collected in Japan, instead Ts collected from China and Korea to which $\mathrm{Tb}$ has not been distributed were used (Murakami, 1997).

A Torymus parasitoid obtained from a gall of Celticecis japonica (Diptera, Cecidomyiidae) on a hackberry, Celties sinensis var. japonica in Matsudo, Chiba Pref. was used as one of the outgroups ("Torymus sp. 1" in Table 1 and Fig. 2). This Torymus sp. 1 was different from any other hitherto known Torymus species (Grissell, 1995) and was determined by Dr. Kamijo to be an "undescribed species" (personal communication).

Definition of Ts, $\mathrm{Tb}$ and unidentified Torymus. The $\mathrm{O} / \mathrm{T}$ ratio was calculated for each parasitoid, except for Torymus sp. 1, in the same manner as that described by Yara et al. (2000). According to the frequency distribution of the $\mathrm{O} / \mathrm{T}$ ratios described by Moriya et al. (1992) and in Moriya's unpublished data, Torymus parasitoids with small O/T ratios categorized as " $\mathrm{S}$ " $(1.03 \leqq \mathrm{O} / \mathrm{T} \leqq 1.29)$ and those with large $\mathrm{O} / \mathrm{T}$ ratios categorized as " $\mathrm{L}$ " $(1.38 \leqq \mathrm{O} / \mathrm{T} \leqq 1.71)$ were classified as $\mathrm{Tb}$ and $\mathrm{Ts}$, respectively. For some individuals whose $\mathrm{O} / \mathrm{T}$ ratios were outside the ranges of the S- or L-categories, three different categories were used: extra small $\mathrm{O} / \mathrm{T}$ ratios were categorized as "XS" $(\mathrm{O} / \mathrm{T}<1.03)$, medium $\mathrm{O} / \mathrm{T}$ ratios were categorized as " $\mathrm{M}$ " $(1.29<\mathrm{O} / \mathrm{T}<1.38)$, and extra large $\mathrm{O} / \mathrm{T}$ ratios were categorized as "XL" $(1.71<\mathrm{O} / \mathrm{T})$ (Table 1$)$. Torymus parasitoids in these three categories were treated as "unidentified Torymus" in this study.

Some individuals from Hebei Province, Liao-

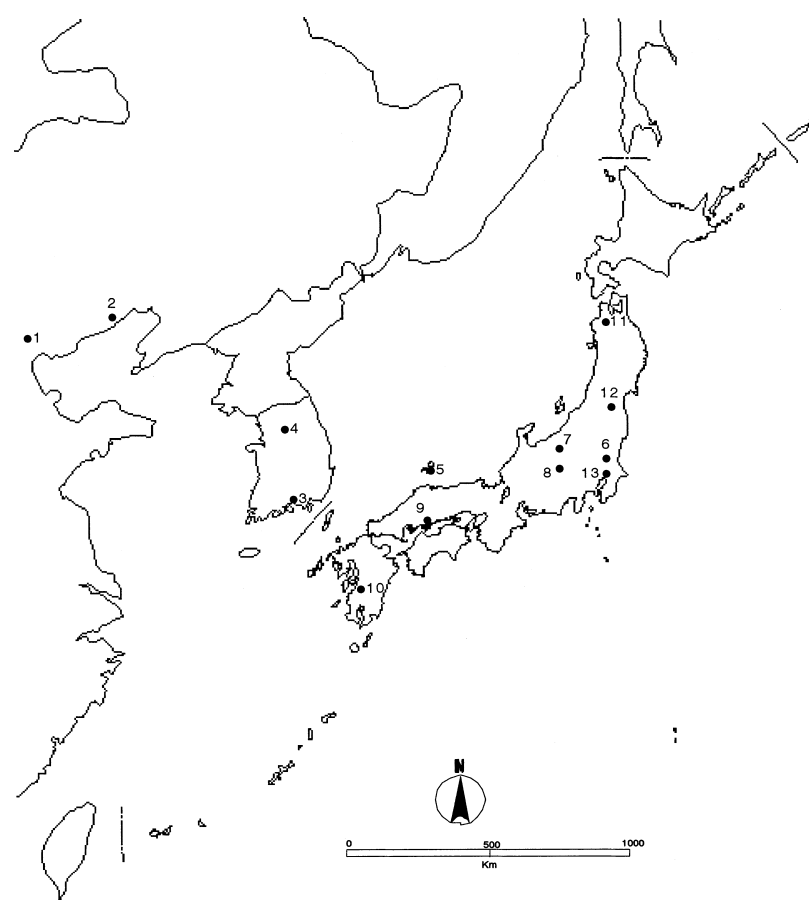

Fig. 1. Collection sites of Torymus spp. used in this study. 1: Zunhua (Hebei Province), 2: Xingcheng (Liaoning Province), 3: Jinyang (Kyongsangnam-do), 4: Chunchon (Kangwon-do), 5: Saigo (Oki Is.), 6: Kukizaki, 7: Obuse, 8: Takamori, 9: Akitsu, 10: Ue, 11: Hirosaki, 12: Nihonmatsu, 13: Matsudo. The numbers correspond to those in Table 1. 
Table 1. Collection information for Torymus specimens used in the study

\begin{tabular}{|c|c|c|c|c|c|c|}
\hline Species & Country & Localities $^{\mathrm{a}}$ & & Emergence period & $\mathrm{O} / \mathrm{T}^{\mathrm{b}}$ & $\mathrm{N}$ \\
\hline \multicolumn{7}{|c|}{ T. sinensis } \\
\hline & China & Zunhua, Hebei Province & (1) & Mar.-Apr. 1993 & $\mathrm{~L}$ & 3 \\
\hline & Korea & Jinyang, Kyongsangnam-do & (3) & Apr. 1992 & $\mathrm{~L}$ & 3 \\
\hline & Korea & Chunchon, Kangwon-do & (4) & Apr. 2000 & $\mathrm{~L}$ & 3 \\
\hline & Japan & Saigo, Shimane Pref. (Oki Is.) & (5) & Apr. 1996 & $\mathrm{~L}$ & 3 \\
\hline \multicolumn{7}{|c|}{ T. beneficus (early-spring strain) } \\
\hline & Japan & Kukizaki, Ibaraki Pref. & (6) & Mar.-Apr. 1988 & $\mathrm{~S}$ & 17 \\
\hline \multicolumn{7}{|c|}{ T. beneficus (late-spring strain) } \\
\hline & Japan & Kukizaki, Ibaraki Pref. & (6) & Apr. 1988 & $\mathrm{~S}$ & 9 \\
\hline \multicolumn{7}{|c|}{ T. beneficus (autumn strain) } \\
\hline & Japan & Nihonmatsu, Fukushima Pref. & $(12)$ & Oct.-Nov. 1990 & $\mathrm{~S}$ & 2 \\
\hline \multicolumn{7}{|c|}{ T. beneficus (emerging in spring) } \\
\hline & Japan & Obuse, Nagano Pref. & (7) & Apr. 1993 & $\mathrm{~S}$ & 4 \\
\hline & Japan & Takamori, Nagano Pref. & (8) & Apr. 1997 & $\mathrm{~S}$ & 3 \\
\hline & Japan & Akitsu, Hiroshima Pref. & (9) & Mar. 1991 & $\mathrm{~S}$ & 2 \\
\hline & Japan & Ue, Kumamoto Pref. & (10) & Mar.-Apr. 1993 & $\mathrm{~S}$ & 3 \\
\hline & Japan & Hirosaki, Aomori Pref. & (11) & Mar. 1993 & $\mathrm{~S}$ & 1 \\
\hline & Japan & Nihonmatsu, Fukushima Pref. & (12) & Mar. 1991 & $\mathrm{~S}$ & 3 \\
\hline \multicolumn{7}{|c|}{ Unidentified Torymus ${ }^{\mathrm{c}}$} \\
\hline & China & Zunhua, Hebei Province & (1) & Apr. 1993 & M & 3 \\
\hline & China & Zunhua, Hebei Province & (1) & Mar.-Apr. 1993 & $\mathrm{~S}$ & 3 \\
\hline & China & Xingcheng, Liaoning Province & (2) & Apr. 1993 & M & 1 \\
\hline & China & Xingcheng, Liaoning Province & (2) & Apr. 1993 & $\mathrm{~S}$ & 1 \\
\hline & Korea & Chunchon, Kangwon-do & (4) & Apr. 2000 & $\mathrm{~S}$ & 3 \\
\hline & Japan & Kukizaki, Ibaraki Pref. & (6) & Mar.-Apr. 1988 & $\mathrm{M}$ & 2 \\
\hline & Japan & Kukizaki, Ibaraki Pref. & (6) & Mar. 1988 & $\mathrm{XS}$ & 4 \\
\hline & Japan & Hirosaki, Aomori Pref. & (11) & Mar. 1993 & $\mathrm{XS}$ & 1 \\
\hline & Japan & Hirosaki, Aomori Pref. & (11) & Apr. 1993 & $\mathrm{XL}$ & 1 \\
\hline & Japan & Nihonmatsu, Fukushima Pref. & (12) & Nov. 1991 & $\mathrm{XS}$ & $1^{\mathrm{d}}$ \\
\hline \multicolumn{7}{|c|}{ Torymus sp. $1^{\mathrm{e}}$} \\
\hline & Japan & Matsudo, Chiba Pref. & (13) & May 2000 & - & 1 \\
\hline
\end{tabular}

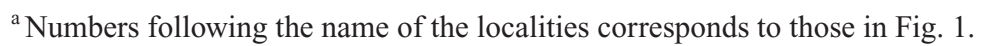

${ }^{\mathrm{b}} \mathrm{O} / \mathrm{T}$ category according to Moriya et al. (1992) and Moriya's unpublished data. XS: $\mathrm{O} / \mathrm{T}<1.03, \mathrm{~S}: 1.03 \leqq 0 / \mathrm{T} \leqq 1.29, \mathrm{M}$ : $1.29<\mathrm{O} / \mathrm{T}<1.38, \mathrm{~L}: 1.38 \leqq \mathrm{O} / \mathrm{T} \leqq 1.71, \mathrm{XL}: 1.71<\mathrm{O} / \mathrm{T}$. See also text for details.

${ }^{\mathrm{c}}$ Specimens that could not be identified according to the $\mathrm{O} / \mathrm{T}$ category and literature on distribution of Torymus wasps (Kamijo, 1982; Murakami, 1997).

${ }^{\mathrm{d}}$ Emerging in autumn.

${ }^{\mathrm{e}}$ A parasitoid of Celticecis japonica (Diptera, Cecidomyiidae) obtained from galls of a leaf of Celtis sinensis var. japonica.

ning Province, and Kangwon-do were treated as unidentified Torymus parasitoids in spite of their Scategory $\mathrm{O} / \mathrm{T}$ ratios, since $\mathrm{Tb}$ is believed not to be distributed in China or Korea (Murakami, 1997).

The three individuals from Saigo (Oki Islands) with L-category $\mathrm{O} / \mathrm{T}$ ratios used in this study were the samples that Toda et al. (2000) classified as Ts by the $\mathrm{O} / \mathrm{T}$ ratio and genotype of malic enzyme. They concluded these Ts must have been present on the islands for a long time before introduced Ts were released in Tottori Pref. in 1991. Thus, the three Ts individuals were treated as "indigenous
Ts" in this study, to distinguish them from Ts being released at various sites in Japan as an introduced natural enemy.

The emergence periods of $\mathrm{TbE}$ and $\mathrm{TbL}$ do not overlap (Moriya et al., 1989), but the periods vary to some extent among years and sites. In one case, for example, $\mathrm{TbE}$ emerged from the latter half of March to the first half of April and TbL emerged from late April to early May (Moriya et al., 1989). Twenty-six Tb parasitoids collected from Kukizaki, Ibaraki Pref. in 1988 were divided into two types because of their two different emergence periods in 
spring: 17 individuals were from March 16 to April 12 and 9 individuals were from April 27 to April 30 in 1988. The former and the latter types were treated as $\mathrm{TbE}$ and $\mathrm{TbL}$, respectively. Other $\mathrm{Tb}$ parasitoids collected in spring were from different years and sites, and therefore their emergence types could not be determined. These Tb parasitoids were therefore tentatively treated as springemergent $\mathrm{Tb}$. $\mathrm{Tb}$ parasitoids collected from Nihonmatsu in autumn were treated as TbA.

DNA extraction, PCR, and sequencing. DNA was extracted from a single adult using an Easy$\mathrm{DNA}^{\mathrm{TM}}$ Kit (Invitrogen) according to the manufacturer's instructions. One hundred microliters of DNA solution were finally obtained. PCR was performed in $100 \mu 1$ reaction volumes using TaKaRa Ex Taq $^{\mathrm{TM}}$ (Takara Shuzo). Two primers, C1-J-1718 (5'-GGAGGATTTGGAAATTGATTAGTTCC-3') and C1-N-2191 (5'-CCCGGTAAAATTAAAATATAAACTTC-3') (Simon et al., 1994) were used. The cycling conditions were as follows: samples were preheated at $94^{\circ} \mathrm{C}$ for $1 \mathrm{~min}$, followed by 35 cycles at $94^{\circ} \mathrm{C}$ for $30 \mathrm{~s}, 45^{\circ} \mathrm{C}$ for $30 \mathrm{~s}$, and $72^{\circ} \mathrm{C}$ for 1 min in a Gene Amp ${ }^{\mathrm{TM}}$ PCR System 9600 (PerkinElmer). Target products (a fragment of COI, ca. 473 bp) were purified using a QIAquick ${ }^{\mathrm{TM}}$ PCR Purification Kit (Qiagen) and both strands directly sequenced using a Thermo Sequenase ${ }^{\mathrm{TM}}$ II dye terminator cycle sequencing kit (Amersham Pharmacia) on an ABI 373 automated sequencer (Applied Biosystems).

Based on the DNA sequences obtained, a new primer, coiT2 (5'-TCATTACCTGTTTTAGCAGG$\left.3^{\prime}\right)$ was designed. To obtain an additional fragment of COI, PCR was performed using a primer pair of coiT2 and TL2-N-3014 (5'-TCCAATGCACTAATCTGCCATATTA-3') (Simon et al., 1994). PCR products (ca. $936 \mathrm{bp}$ ) were sequenced directly in the same manner.

Phylogenetic analysis of sequence data. The sequence data were aligned using Clustal W (Thompson et al., 1994). Neighbor-joining (NJ) analyses using Kimura distances (Kimura, 1980) were performed using PAUP* 4.0 beta 10 (Swofford, 2000). Torymus sp. 1 and Apis mellifera (accession number L06178) were used as outgroups. The support for each node was assessed by bootstrapping with 500 replications performed.

\section{RESULTS AND DISCUSSION}

For COI, 1,129 bp of nucleotide coding regions were determined (DDBJ/EMBL/GenBank, accession numbers AB070473 to AB070504). A total of 143 sites on the nucleotide regions were polymorphic. Thirty-one haplotypes were found among the individuals investigated. There were three distinct groups in the phylogenetic tree obtained: "Group A", "Group B" and "Group C". Group A consisted of three subgroups, "subgroup 1", "subgroup 2" and "subgroup 3" (Fig. 2).

First, the genetic relationships among Ts and the three strains of $\mathrm{Tb}(\mathrm{TbE}, \mathrm{TbL}$, and $\mathrm{TbA})$ are discussed. TbE (17 individuals collected from Kukizaki, highlighted in green in Fig. 2) had two haplotypes and belonged to Group B in the phylogenetic tree. TbA (two from Nihonmatsu, highlighted in yellow) had two haplotypes and also belonged to Group B. Of these individuals seven TbE (Kukizaki) and one $\mathrm{TbA}$ (Nihonmatsu) showed the same haplotype. These results indicate that $\mathrm{TbE}$ and $\mathrm{TbA}$ are not fully genetically differentiated in spite of the differences in ecological traits (emergence period). On the other hand, Ts (three from Hebei Province, three from Kyongsangnam-do, three from Kangwon-do, and three from Oki Is., highlighted in pink) had five haplotypes and belonged to subgroup 1 and subgroup 2 of Group A. TbL (nine from Kukizaki, highlighted in red) had four haplotypes and belonged to subgroup 3 of Group A, suggesting that $\mathrm{TbL}$ is more closely related to Ts considered as the different species than to TbE and $\mathrm{TbA}$ strains considered as the same species. This view is consistent with the fact that there are several isozyme markers that discriminate between $\mathrm{TbE}$ and $\mathrm{TbL}$, or between $\mathrm{Ts}$ and $\mathrm{TbE}$, whereas no isozyme marker to discriminate between Ts and TbL has yet been found (Izawa et al., 1992, 1996; Noda et al., unpublished data). It is clearly necessary to revise the taxonomical treatment of Ts and the three strains of $\mathrm{Tb}$, especially TbL (Kamijo, 1982; Murakami, 1997).

The results of this study make it possible to determine the phylogenetic position of the "unidentified Torymus" collected from China and Korea, which could not be identified by $\mathrm{O} / \mathrm{T}$ ratio. As described above, Ts formed two genetically different subgroups, i.e., subgroup 1 and subgroup 2 of Group A (Fig. 2). In subgroup 1, six individuals of 


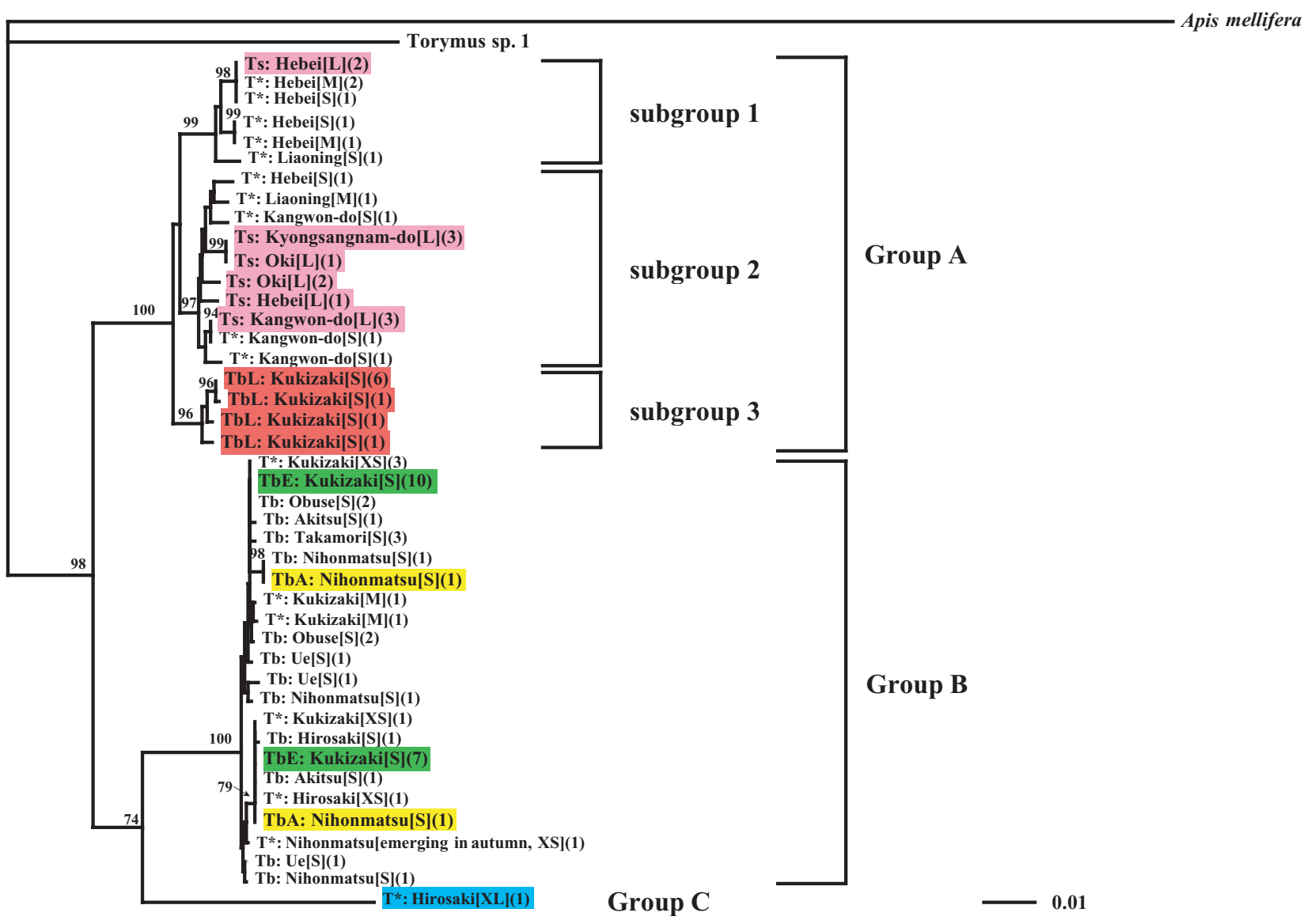

Fig. 2. Phylogenetic tree inferred from $1,129 \mathrm{bp}$ sequences of the mitochondrial COI gene of Torymus spp. using the neighborjoining method. Apis mellifera and Torymus sp. 1 were used as outgroups. The abbreviations for each species and strain are as follows; Ts: Torymus sinensis (highlighted in pink), TbE: early-spring strain of T. beneficus (highlighted in green), TbL: late-spring strain of T. beneficus (highlighted in red), TbA: autumn strain of T. beneficus (highlighted in yellow), Tb: T. beneficus emerging in spring (strain unidentified), $\mathrm{T}^{*}$ : unidentified Torymus. One unidentified Torymus from Hirosaki with the XL-category O/T ratio is highlighted in blue. $\mathrm{O} / \mathrm{T}$ categories are indicated in square brackets. The number of specimens are given in parentheses. Bootstrap probabilities (500 replicates) are provided on each branch when they are greater than $70 \%$. The scale of distances is shown under the tree.

unidentified Torymus collected from China (five from Hebei Province and one from Liaoning Province in the S- or M-category $\mathrm{O} / \mathrm{T}$ ratios) were found. Of these individuals three "unidentified" specimens from Hebei Province had the same haplotype, as did two Ts from Hebei Province. Thus, we can say from the genetic viewpoint that these unidentified Torymus were Ts, in spite of their smaller $\mathrm{O} / \mathrm{T}$ ratios. Similarly, five other unidentified Torymus collected from China and Korea (one from Hebei Province, one from Liaoning Province and three from Kangwon-do with the S- or M-category $\mathrm{O} / \mathrm{T}$ ratios) can be regarded as $\mathrm{Ts}$ because they belong to subgroup 2. Furthermore, these two subgroups appear to be different from each other in collection site: subgroup 1 consisted of Ts collected from China only, whereas subgroup 2 consisted of parasitoids from China, Korea and Japan (Oki Is.). This prompts an interesting question as to whether the latter is distributed more widely than the former. However, further investigation is needed to answer this question.

We were also able to determine the phylogenetic position of the $\mathrm{Tb}$ parasitoids and most of the unidentified Torymus that could not be identified by $\mathrm{O} / \mathrm{T}$ ratio and emergence period collected from Japan. Although the spring-emerging $\mathrm{Tb}$ specimens (four from Obuse, three from Takamori, two from Akitsu, three from Ue, one from Hirosaki and three from Nihonmatsu) had not had their emer- 
gence types (TbE or TbL) determined, all of them belonged to Group B (Fig. 2). These individuals could be regarded as TbE because Group B included TbE and TbA. The unidentified Torymus emerging in spring (six from Kukizaki in the XSor M-category and one from Hirosaki in the XScategory $\mathrm{O} / \mathrm{T}$ ratios) and those emerging in autumn (one from Nihonmatsu in the XS-category $\mathrm{O} / \mathrm{T}$ ratio) also belonged to Group $\mathrm{B}$. Thus, it is conceivable that the former and the latter were $\mathrm{TbE}$ and $\mathrm{TbA}$, respectively. However, we cannot genetically identify one unidentified Torymus with the XL-category $\mathrm{O} / \mathrm{T}$ ratio collected from Hirosaki (highlighted in blue in Fig. 2). This individual solely formed Group C, indicating an indigenous Torymus parasitoid, which was genetically as well as morphologically different from the $\mathrm{Ts}$ and $\mathrm{Tb}$ strains, exploited galls of D. kuriphilus in Japan. Further investigation will be needed to genetically identify the unidentified Torymus parasitoids.

The $\mathrm{O} / \mathrm{T}$ ratio has up to now been used as a good indicator for simply discriminating between Ts and $\mathrm{Tb}$, though it has not identified several Torymus parasitoids exploiting D. kuriphilus galls in chestnut trees. The present results contradict the general belief that $\mathrm{Ts}$ has a larger $\mathrm{O} / \mathrm{T}$ ratio while $\mathrm{Tb}$ has a smaller one (Kamijo, 1982; Murakami, 1997), categorized respectively as L- and $\mathrm{S}-\mathrm{O} / \mathrm{T}$ ratio in this study. As described above, the unidentified Torymus from China and Korea can be treated as Ts in the phylogenetic tree, in spite of their different $\mathrm{O} / \mathrm{T}$ ratios categorized as $\mathrm{M}$ or $\mathrm{S}$. In addition, the unidentified Torymus from Japan can be regarded as $\mathrm{TbE}$ or $\mathrm{TbA}$, in spite of their $\mathrm{O} / \mathrm{T}$ ratios categorized as $\mathrm{M}$ or XS. These results indicate that the $\mathrm{O} / \mathrm{T}$ ratio is not a reliable marker for discrimination between $\mathrm{Ts}$ and $\mathrm{Tb}$. Much more accurate information is likely to be obtained by using COI and other reliable molecular markers to investigate the ecological interactions of Torymus as well as their genetic relationships (Yara et al., 2000).

\section{ACKNOWLEDGEMENTS}

This paper owes much to the helpful comments and technical suggestions of Dr. M. Muraji (National Institute of Agrobiological Sciences). I am deeply indebted to Drs. M. Shiga (Society for Techno-innovation of Agriculture, Forestry and Fisheries), I. Adachi (National Institute of Fruit Tree Science), S. Moriya (National Agricultural Research Center), Y. Shirai (National Institute for Agro-Environmental Sciences), J. K. Kim (College of Forest Sciences, Kangwon National Univer- sity), S. Toda (National Institute of Fruit Tree Science), Y. Gyoutoku (Kumamoto Prefectural Agricultural Research Center), T. Sasawaki (Nagano Fruit-tree Experiment Station), and $\mathrm{K}$. Tabuchi (Chiba University) for providing me with the samples. Special thanks are also due to Drs. K. Kamijo (Bibai City) and K. Konishi (National Agricultural Research Center for Hokkaido Region) for the identification of the specimens. I would like to thank Drs. K. Kamijo and T. Shimoda (National Agricultural Research Center) for their helpful comments and suggestions on the draft. Thanks are also due to Dr. N. Hinomoto (National Institute of Agrobiological Sciences) for support in the experiments. I appreciate the two anonymous referees' comments that improved this paper immensely. This study was supported in part by a Grant-in-Aid (Bio Cosmos Program) from the Ministry of Agriculture, Forestry and Fisheries, Japan.

\section{REFERENCES}

Caterino, M. S., S. Cho and F. A. H. Sperling (2000) The current state of insect molecular systematics: a thriving Tower of Babel. Annu. Rev. Entomol. 45: 1-54.

Grissell (1995) Toryminae (Hymenoptera: Chalcidoidea: Torymidae): a Redefinition, Generic Classification, and Annotated World Catalog of Species. Memoirs on Entomology, International Volume 2. Associated Publishers, Gainesville, Florida. 470 pp.

Izawa, H., Mh. Osakabe and S. Moriya (1992) Isozyme discrimination between an imported parasitoid wasp, Torymus sinensis Kamijo and its sibling species, T. beneficus Yasumatsu et Kamijo (Hymenoptera: Torymidae), attacking Dryocosmus kuriphilus Yasumatsu (Hymenoptera: Cynipidae). Jpn. J. Appl. Entomol. Zool. 36: 58-60 (in Japanese with English summary).

Izawa, H., Mh. Osakabe and S. Moriya (1995) Relation between banding patterns of malic enzyme by electrophoresis and a morphological character in exotic and native Torymus species. Appl. Entomol. Zool. 30: 37-41.

Izawa, H., Mh. Osakabe, S. Moriya and S. Toda (1996) Use of malic enzyme to detect hybrids between Torymus sinensis and T. beneficus (Hymenoptera: Cynipidae) attacking Dryocosmus kuriphilus (Hymenoptera: Cynipidae) and possibility of natural hybridization. Jpn. J. Appl. Entomol. Zool. 40: 205-208 (in Japanese with English summary).

Kamijo, K. (1982) Two new species of Torymus (Hymenoptera, Torymidae) reared from Dryocosmus $k u-$ riphilus (Hymenoptera, Cynipidae) in China and Korea. Kontyû (Tokyo) 50: 505-510.

Kimura, M. (1980) A simple method for estimating evolutionary rate of base substitutions through comparative studies of nucleotide sequences. J. Mol. Evol. 16: $111-120$

Moriya, S., Inoue, K. and Mabuchi, M. (1989) The use of Torymus sinensis to control chestnut gall wasp, Dryocosmus kuriphilus, in Japan. FFTC Techn. Bull. 118: 1-12.

Moriya, S., K. Inoue, M. Shiga and M. Mabuchi (1992) Interspecific relationship between an introduced parasitoid, Torymus sinensis Kamijo, as a biological control agent of 
the chestnut gall wasp, Dryocosmus kuriphilus Yasumatsu, and an endemic parasitoid, Torymus beneficus Yasumatsu et Kamijo. Acta Phytopathol. Entomol. Hungarica 27: 479-483.

Murakami, Y. (1981) The parasitoids of Dryocosmus kuriphilus Yasumatsu (Hymenoptera: Cynipidae) in Japan and the introduction of a promising natural enemy from China (Hymenoptera: Chalcidoidea). J. Fac. Agr., Kyushu Univ. 25: 167-174.

Murakami, Y. (1988) Ecotypes of Torymus (Syntomaspis) beneficus Yasumatsu et Kamijo (Hymenoptera: Torymidae) with different seasonal prevalence of adult emergence. Appl. Entomol. Zool. 23: 81-87.

Murakami, Y. (1997) Natural Enemies of the Chestnut Gall Wasp, Approaches to Biological Control. Kyusyu University Press, Fukuoka. 308 pp. (in Japanese).

Ôtake, A. (1987) Comparison of some morphological characters among two strains of Torymus beneficus Yasumatsu et Kamijo and T. sinensis Kamijo (Hymenoptera: Torymidae). Appl. Entomol. Zool. 22: 600-609.

Shirai, Y., I. Adachi and S. Moriya (1999) Records of release of Torymus sinensis Kamijo (Hymenoptera: Torymidae), an introduced biological control agent of chestnut gall wasp in Japan. Bull. Natl. Inst. Fruit Tree Sci. 33:
163-178 (in Japanese with English summary).

Simon, C., F. Frati, A. Beckenbach, B. Crespi, H. Liu and P. Flook (1994) Evolution, weighting, and phylogenetic utility of mitochondrial gene sequences and a compilation of conserved polymerase chain reaction primers. Ann. Entomol. Soc. Am. 87: 651-701.

Swofford, D. L. (2000) PAUP*. Phylogenetic Analysis Using Parsimony and Other Methods. Version 4. Sinauer, Sunderland, Massachusetts.

Thompson, J. F., F. G. Higgins and T. G. Gibson (1994) CLUSTAL W: Improving the sensitivity of progressive multiple sequence alignment through sequence weighting, positions-specific gap penalties and weight matrix choice. Nucleic Acids Res. 22: 4673-4680.

Toda, S., M. Miyazaki, Mh. Osakabe and S. Komazaki (2000) Occurrence and hybridization of two parasitoid wasps, Torymus sinensis Kamijo and T. beneficus Yasumatsu et Kamijo (Hymenoptera: Torymidae) in the Oki islands. Appl. Entomol. Zool. 35: 151-154.

Yara, K., E. Yano, T. Sasawaki and M. Shiga (2000) Detection of hybrids between introduced Torymus sinensis and native $T$. beneficus (Hymenoptera: Torymidae) in central Japan, using malic enzyme. Appl. Entomol. Zool. 35: 201-206. 